\title{
Escenarios móviles y centros de contacto, una experiencia significativa en la dinamización del apoyo social
}

\author{
Mobile scenarios and contact centers, a significant experience in the invigoration of social support
}

\begin{abstract}
Jonnathan Narvaez ${ }^{1}$
1 Psicólogo, Lic. en Filosofía, Pensamiento Político y Económico. Especialista en Estudios Latinoamericanos. Magíster en Investigación Integrativa. Docente Programa de Psicología, Integrante Grupo de Investigación Libres Pensadores Universidad de Nariño. Pasto, Colombia. e-mail: jonnathanhar@hotmail.com
\end{abstract}

\section{Resumen}

Introducción: El presente artículo presenta los resultados del eje de apoyo social de una propuesta de intervención multinivel desarrollada en la Comuna 5 del Municipio de Buenaventura en el pacifico colombiano, focalizada en la dinamización comunitaria de los escenarios móviles y los centros de contacto como estrategias comunitarias para la articulación de los servicios sociales y el fortalecimiento de redes colaborativas de acción y apoyo social. Objetivo: Determinar la influencia de los escenarios móviles y centros de contacto en la dinamización del apoyo social. Materiales y métodos: En este artículo se presentan los resultados de una investigación cualitativa de enfoque crítico social, las técnicas de investigación fueron la asamblea participativa y los talleres de investigación participativa las cuales se desarrollaron desde cuatro ejes de intervención multinivel trasversales a la experiencia de acuerdo a los contenidos del apoyo social y la ejecución de las estrategias de activación comunitaria: escenarios móviles y centros de contacto. Resultados: Entre los principales resultados se presenta la articulación de los ejes de apoyo social informacional, emocional, instrumental y evaluativo en función del fortalecimiento del capital social en el desarrollo de las capacidades comunitarias conducentes a la estructuración de redes de acción y gestión colectiva; asimismo se identifica la pertinencia de los escenarios móviles y centros de contacto como estrategias para la dinamización del apoyo social y la activación comunitaria. Conclusiones: La estrategia de escenarios móviles y centros de contacto se convierte en una innovadora alternativa para la promoción de la participación ciudadana en la dinamización del apoyo social, garantizando no sólo mayor accesibilidad y equidad en la prestación de servicios, sino también el desarrollo del capital social en torno a las capacidades comunitarias locales. Asimismo, el apoyo social permite a las comunidades en ambientes sociales de pobreza, violencia y desintegración promover la autoestima colectiva y la solidaridad efectiva, como atenuantes de estresores sociales marcados por el individualismo y la exclusión social.

Palabras clave: Apoyo social; centros de contacto; escenarios móviles; intervención comunitaria. (Fuente: DeCS, Bireme).

\begin{abstract}
Introduction: This article presents the results of the axis of social support of a proposed multi-level intervention developed in the Commune 5 of the Municipality of Buenaventura on the Colombian Pacific, focused on community revitalization of mobile scenarios and contact centers as community strategies coordination of social services and
\end{abstract}


strengthening collaborative networks of action and social support. Objective: To determine the influence of mobile scenarios and contact centers in the revitalization of social support. Materials and methods: This article presents the results of a qualitative research with critical social approach; the research techniques were the participatory assembly and participatory research workshops which were developed from four areas of multilevel intervention transverse to the experience according to the contents of social support and the implementation of the community activation strategies: mobile scenarios and contact centers. Results: Among the main results, the articulation of social support informational, emotional, instrumental and evaluative based on the strengthening of social capital in the development of community capabilities leading to the structuring of action networks and collective management is presented as well as the relevance of mobile scenarios and contact centers as strategies for the revitalization of social support and community activation is identified. Conclusions: The strategy of mobile scenarios and contact centers has become an innovative alternative to promote citizen participation in the revitalization of social support, ensuring not only greater accessibility and equity in the provision of services but also the development of capital social surrounding local community capacities. Also, social support allows communities in social environments of poverty, violence and disintegration to promote collective self-esteem and effective solidarity as a mitigating social stressors marked by individualism and social exclusion.

Keywords: Social support; contact centers; mobile scenarios; community intervention. (Source: DeCS, Bireme).

\section{Introducción}

Buenaventura corresponde el principal puerto marítimo de la nación sobre el pacífico donde se embarca el $60 \%$ de las exportaciones del país. Sin embargo, estadísticos del DANE ${ }^{1}$, reportan que el $50 \%$ de los habitantes del puerto viven en condiciones de pobreza. Más alarmantes son los resultados de una encuesta de pobreza por ingresos realizada en 2004 la cual concluyó que el $80.6 \%$ de las familias vivía en situación de pobreza y de ellas $43.5 \%$ de indigencia ${ }^{2}$. Bajamar es un importante sector del puerto construido sobre las aguas del mar Pacífico y diversos riachuelos, el cual progresivamente se fue poblando cuando varias familias buscaron asentarse en lugares cercanos a la marea con la finalidad de permanecer cerca de las fuentes de pesca, principal medio de subsistencia.

La comuna 5 comprende los barrios Pascual de Andagoya, La Palera, Punta del Este, Santa Cruz, La Inmaculada, Santa fe y Miramar, se estima alberga cerca 51 mil personas, en su gran mayoría afrodescendientes procedentes de los departamentos del Choco, Nariño, Cauca y Valle quienes migraron de sus poblados de origen como consecuencia del conflicto armado y el desplazamiento forzado. Según información de la Fundación Rostros y Huellas del Sentir Humano ${ }^{3}$ el poblamiento de esta zona comenzó hace más de 40 años, cuando muchas familias abandonaron sus comunidades ancestrales en las cuencas de los ríos de Buenaventura, o en el litoral pacífico motivados por la búsqueda de mejores condiciones de vida como el acceso a la educación y el empleo, terminando hacinadas en un sector del puerto, desolados por la violencia, la pobreza, la falta de oportunidades y el abandono gubernamental.

El contexto inicial de la experiencia desde un diagnóstico preliminar evidenciaba difíciles condiciones de habitabilidad. Entre los factores generales incidentes se encontró la poca mediación del Estado colombiano en la garantía de derechos fundamentales, el difícil acceso a los servicios sociales dada la insuficiente cobertura y la inoperancia institucional, en conjunto con crecientes factores de riesgo hacia el consumo de sustancias psicoactivas, delincuencia juvenil y desnutrición infantil; condiciones permeadas por prácticas sociales de intimidación y miedo como efecto del control social de grupos armados ilegales con influencia en la zona ${ }^{4}$. Bajo este panorama, y con el fin de consolidar y dinamizar escenarios móviles y centros de contacto se siguió el modelo de apoyo social, el cual propone la construcción de sistemas adecuados de colaboración, por medio de la creación de grupos de apoyo y autoayuda, cuya eficacia contribuye a satisfacer necesidades humanas como las de seguridad, contacto social, pertenencia, estima y afecto ${ }^{5}$. 
El apoyo social dentro los escenarios móviles y centros de contacto, constituye un componente fundamental para el desarrollo del bienestar individual, familiar, y social impactando de forma positiva al desarrollo de recursos locales y la integración comunitaria. En el fortalecimiento del apoyo social, Gottlieb ${ }^{6}$ señala cinco niveles entre los que se destacan la estructura social referente a la modificación del ambiente físico y sociocultural en el que se desenvuelve la persona, en donde se modifica la estructura y las funciones de los lazos sociales, desde aspectos como la política social, las normas, roles, y dinámicas interaccionales; y la estructura comunitaria, en donde, se busca promover la confianza pública en las fuentes informales de ayuda y estimular la expresión de apoyo en la vida cotidiana, componente que explica la vinculación de los servicios sociales dentro de las redes informales de apoyo social que garanticen el desarrollo comunitario y la promoción humana de los agentes destinatarios.

Al respecto, Manfred Max-Neef ${ }^{7}$ señala que el desarrollo a escala humana, "se sustenta en la satisfacción de las necesidades humanas fundamentales, en la generación de niveles crecientes de auto dependencia y en la articulación orgánica de los seres humanos con la naturaleza y la tecnología, de los procesos globales con los comportamientos locales, de lo personal con lo social, de la planificación con la autonomía y de la Sociedad Civil con el Estado" siendo los servicios sociales una importante herramienta de la política social encargada de proveer a las mayorías excluidas de los mínimos fundamentales para el desarrollo personal y el bienestar social; Colmenares ${ }^{8}$ señala a los servicios sociales como el cuarto pilar de los sistemas de bienestar y apoyo social en una sociedad, dirigidos a solventar las necesidades educativas y de integración comunitaria, como efecto de la articulación de las instituciones gubernamentales y civiles con los habitantes de zonas históricamente marginadas.

Los centros de contacto por su parte, constituyen dentro de la experiencia, un servicio comunitario orientado a la facilitación de herramientas psicosocioeducativas destinadas al fortalecimiento de comunidades protectoras y la mejora progresiva de la percepción del apoyo social percibido. La estrategia de centro de contacto no está dirigida a suplir necesidades fisiológicas, sino a fortalecer la alianza entre instituciones y comunidades, desde el desarrollo de procesos organizativos, el asesoramiento profesional, el direccionamiento de situaciones problema y la generación de acciones comunitarias. Lo anterior, en coherencia con el postulado de Durá y Garcés ${ }^{9}$, quienes definen el apoyo social como "una transacción real entre dos o más personas, en la que se da una implicación emocional, una ayuda instrumental, información y valoración"; por lo tanto, se da un intercambio individuo - comunidad, con el objetivo de proveer o recepcionar ayuda, con el fin de incrementar el bienestar tanto individual como comunitario.

En este contexto, y de acuerdo con Herrera ${ }^{10}$ los escenarios móviles permiten una intervención más focalizada desde la sectorización barrial en unidades micro sociales, donde diversos agentes asistenciales de manera multidisciplinaria recepcionan y direccionan las necesidades educativas, sociales, de salud, jurídicas y familiares de las comunidades destinatarias a fin focalizar los casos y fortalecer los lazos vinculares nacientes entre Institución - comunidad. Dicha estrategia permite una transacción real entre estructuras sociales y comunitarias, en donde se proporciona un apoyo de carácter informacional, emocional, instrumental y evaluativo, con el objetivo de consolidar escenarios móviles como centros de apoyo comunitario que provean diversos niveles de apoyo social en la comunidad.

De allí, que los centros de contacto al articular a escenarios móviles, se constituyen en epicentros de apoyo social popular y multisectorial ${ }^{10}$, entre tanto posibilitan el acceso a diversos servicios profesionales desde el marco de la orientación social o el seguimiento de casos, hacia la restitución del derecho a los servicios sociales y el fortalecimiento de la eficacia institucional en el campo del apoyo social percibido y la dinamización comunitaria.

En cuanto a la funcionalidad del apoyo social, a los escenarios móviles y centro de contacto fueron 
articulados los cuatro tipos de apoyo social. El apoyo informacional, se concibió como toda aquella información referente al acceso a los servicios sociales que los habitantes de la comunidad recibieron a través de los centros de contacto. El apoyo emocional, constituyó la comunicación y expresión de afectos y sentimientos facilitados desde escenarios móviles con la finalidad de que los participantes afirmaran su sentido de pertenencia e integración social a la comunidad $^{11}$. A su vez, el apoyo instrumental, permitió a los participantes impulsar conductas de ayuda, que favorecieran el bienestar comunitario y restablecieran la calidad de vida de otros destinatarios dentro y fuera de los centros de contacto. Por último, el apoyo evaluativo, en el ejercicio de la IAP facultó a los agentes comunitarios para la realización del procesos de auto reflexión y autoevaluación con base en la oferta de información sobre los objetivos y procesos de la comunidad.

En el campo del apoyo social, los centros de contacto constituyeron dentro de la experiencia práctica una estrategia diseñada por el equipo interventor, para fomentar servicios comunitarios orientados a la facilitación de herramientas psicosocioeducativas destinadas al fortalecimiento del apoyo social como factor protector de la comunidad. La estrategia de centro de contacto no está dirigida a suplir necesidades fisiológicas, sino a fortalecer la alianza entre instituciones y comunidades, desde el fortalecimiento de procesos organizativos, el asesoramiento profesional, el direccionamiento de situaciones problema y la generación de acciones comunitarias, apoyos emocional, instrumental, evaluativo e informacional.

De acuerdo a Narváez ${ }^{12}$, los escenarios móviles dentro de la experiencia práctica, comprenden para los interventores, una serie de jornadas de atención e inserción comunitaria, donde diversos agentes asistenciales de manera multidisciplinaria, recepcionan y direccionan las necesidades educativas, socio-jurídicas, de salud, y familiares de las comunidades destinatarias a fin focalizar los casos y fortalecer los lazos vinculares nacientes entre Institución comunidad.
Para Herrera ${ }^{10}$ los escenarios móviles permiten una intervención más focalizada desde la sectorización barrial en unidades microsociales, donde se identifica junto con la comunidad las necesidades, soluciones y problemas. De allí, que el presente artículo evidencia como la experiencia de activación comunitaria en la comuna 5 de Buenaventura articula desde la Investigación Acción Participativa la vinculación socioafectiva, el apoyo instrumental y las esferas de solidaridad e integración comunitaria12; donde el contexto, permitió "analizar situaciones comunitarias en los que las comunidades establecen redes vinculares e interaccionales a fin de dinamizar el estatismo de los grupos sociales como destinatarios de políticas asistenciales que vulneran su condición de tránsito ${ }^{13}$ y da cuenta del potencial dinamizador de la comunidad en el fortalecimiento de redes de apoyo y autoayuda a través de escenarios móviles y centros de contacto.

\section{Materiales y métodos}

Se desarrolló la propuesta metodológica de la investigación-acción- participativa (IAP), la cual se basa en la inserción del investigador en la comunidad, el análisis de sus condiciones históricas y estructura social, la promoción participativa del nivel de conciencia de los agentes locales, el desarrollo de organizaciones y grupos de acción con un énfasis en la solución de problemas y el compromiso militante con sus comunidades ${ }^{14}$, lo cual permite al investigador obtener conocimientos colectivos para transformar una determinada realidad social ${ }^{15}$, "al mismo tiempo que interviene, posibilitando la transformación de situaciones problemáticas para los grupos poblacionales con los que acciona" 16 .

La investigación intervención tuvo como objetivo dinamizar fuentes de apoyo social y autoayuda desde la activación de escenarios móviles y centros de contacto en la comuna 5 del distrito de Buenaventura. En consecuencia, se generó un proceso metodológico sistemático, en la implementación de escenarios móviles y centros de contacto que involucró a las comunidades en la producción colectiva de los conocimientos 
necesarios que garanticen la accesibilidad de los pobladores a servicios sociales y maximicen la percepción de apoyo social, desde el fortalecimiento del capital social y cultural de las comunidades destinatarias.

De acuerdo con Ander-Egg17, la IAP supone tres grandes fases, diagnóstico, intervención y evaluación, las cuales no tienen una secuencia lineal, sino más bien circular. De acuerdo a su postulado, la investigación-intervención, siguió las siguientes fases:

a) Diagnóstico: Se dieron los procesos de inserción, reconocimiento de campo y contextualización de la situación problema, en el primer proceso tuvo lugar la inmersión comunitaria de los investigadores en el contexto con la finalidad de establecer relaciones vitales en los entornos naturales de los actores y promover la activación participativa. Una vez establecidos los vínculos con la comunidad y reconocido el espacio ambiental, comunitario y político de la comuna, se levantó información preliminar que permitiera esclarecer las condiciones iniciales del apoyo social y el acceso a los servicios sociales, información con la cual se definió la viabilidad del proyecto y se establecieron los acuerdos éticos y comunitarios para el desarrollo de escenarios móviles y centros de contacto en la Comuna 5.

En cuanto a las condiciones de inserción comunitaria los investigadores canalizaron a agentes locales a través de las redes de cooperación institucionales con información de la Subsecretaria de Desarrollo Comunitario, quienes favorecieron la focalización de líderes y agentes comunitarios con quienes se estableció una agenda de concertación desde el análisis de impacto de la iniciativa sobre las dimensiones familiares y comunitarias. Una vez establecido el contacto inicial se cumple con el protocolo ético, a través de la firma de la autorización de acceso a la comunidad y los consentimientos informados de los líderes comunitarios, posteriormente se socializa la iniciativa con las comunidades e inicia la ejecución del proyecto.

b) Intervención: En ésta fase se aplicó el diseño de intervención multinivel Tabla 1 la cual tuvo como ejes de intervención los apoyos informacional, instrumental, evaluativo y emocional desde la aplicación de las estrategias trasversales a la experiencia, los escenarios móviles y centros de contacto.

c) Evaluación: En los procesos participativos es trasversal a toda gestión comunitaria la evaluación como retroalimentación permanente de la acción, de manera que ésta comprendió una práctica trasversal y permanente en el desarrollo del proyecto. En la evaluación de acuerdo a los postulados del apoyo social se desarrollaron dos estrategias 1) la evaluación desde los actores, la cual se desarrolló a través de la autoevaluación donde cada participante desde su grupo de referencia analizaba el impacto de las estrategias sobre las dimensiones individuales y familiares, y 2) La evaluación desde el proceso, desarrollada a partir del mecanismo de comparación social que permitió monitorear los cambios generados en la dinámicas comunitarias a partir de la implementación de estrategias y procesos proyectados. Esta estrategia en el campo de la investigación participativa favoreció la reflexión sobre la acción al ofrecer insumos para la retroalimentación y modificación de los suministros de activación comunitaria y visibilizar la responsabilidad de la comunidad en la gestión y dirección colaborativa del proyecto.

\section{Técnicas e instrumentos}

Asamblea participativa: Técnica de trabajo de campo que consistió en la participación consiente de la comunidad como grupo social, se desarrolla mediante la incorporación a las diferentes etapas de la intervención de niños, adolescentes y adultos como grupos de interés, tuvo a lo largo del estudio la finalidad de plantear y buscar soluciones colectivas a los problemas comunitarios en la facilitación del apoyo social18.

Taller de investigación participativa: Comprende un conjunto de herramientas articuladas para la dinamización de situaciones sociales desde la participación de la comunidad, la técnica fue utilizada para dinamizar, verificar y complementar la información en función de la organización de escenarios móviles. 
Cartografía social: Es un instrumento de evaluación e intervención la cual permite descripciones objetivas y subjetivas del sujeto en estudio, favoreciendo la construcción de conocimiento integral de un territorio desde la participación y el compromiso social de las comunidades

Participantes. Grupos poblacionales de siete barrios de la comuna 5 del municipio de Buenaventura- Colombia. Participaron en total 1040 personas de acuerdo a la siguiente distribución: 30 Agentes comunitarios y culturales, 400 participantes de la población infanto-juvenil, 300 pertenecientes a adulto joven, 200 personas de adulto medio y 110 de adulto mayor.

Estrategia de intervención. La experiencia estuvo orientada por cuatro ejes de intervención trasversales, dirigidos a la implementación de escenarios móviles de atención y centros de contacto comunitario que permitan la movilización de la base comunitaria entorno al apoyo social en la comuna 5 del municipio de
Buenaventura - Valle. Dicho proceso tuvo lugar desde la inserción comunitaria y la focalización de actores con los cuales se avaló de manera participativa la estrategia. Se propuso un plan de intervención multinivel desde el modelo de apoyo social en la perspectiva de la transitividad comunitaria.

Entre los elementos claves de la propuesta se encuentra la ejecución interdisciplinar de escenarios móviles cuyo objetivo se centró en promover la movilización y participación activa de la población en relación al apoyo instrumental e informacional; y la instalación de un centro de contacto que operativice el apoyo emocional y evaluativo mediante la atención, direccionamiento y resolución de problemáticas personales y psicosociales. La experiencia contó con cuatro ejes de intervención multinivel trasversales de acuerdo a los contenidos del apoyo social, 1) Apoyo instrumental, 2) Apoyo emocional. 3) Apoyo informacional y 4) Apoyo evaluativo; los cuales se articularon a las estrategias de activación comunitaria: escenarios móviles y centros de contacto. (Tabla 1)

Tabla 1. Suministros de activación comunitaria articulación escenarios móviles y centros de contacto - apoyo social

\begin{tabular}{|c|c|c|c|c|}
\hline \multirow{2}{*}{ Eje de intervención } & \multicolumn{4}{|c|}{ Suministros de activación comunitaria } \\
\hline & \multicolumn{2}{|c|}{$\begin{array}{l}\text { Estrategia } \\
\text { escenarios móviles }\end{array}$} & \multicolumn{2}{|c|}{$\begin{array}{c}\text { Estrategia } \\
\text { centros de contacto }\end{array}$} \\
\hline \multirow[t]{2}{*}{ Apoyo instrumental } & \multirow[t]{2}{*}{$\begin{array}{l}\text { Identificación de redes } \\
\text { apoyo naturales } \\
\text { (Diagnóstico) }\end{array}$} & \multicolumn{3}{|c|}{$\begin{array}{l}\text { Implementación de jornadas de atención y movilización } \\
\text { del apoyo comunitario } \\
\text { (Intervención). }\end{array}$} \\
\hline & & \multicolumn{3}{|c|}{$\begin{array}{l}\text { Generación de acciones de apoyo y autoayuda } \\
\text { (Intervención) }\end{array}$} \\
\hline Apoyo informacional & $\begin{array}{l}\text { Socialización de rutas de } \\
\text { atención }\end{array}$ & \multicolumn{3}{|c|}{$\begin{array}{l}\text { Capacitación servicios sociales, derechos y ciudadanía. } \\
\text { (Intervención) }\end{array}$} \\
\hline Apoyo emocional & & \multicolumn{2}{|c|}{$\begin{array}{l}\text { Asistencia profesional de servicios sociales, } \\
\text { direccionamiento de casos y activación de rutas de } \\
\text { atención. } \\
\text { (Intervención). }\end{array}$} & $\begin{array}{l}\text { Implementación de } \\
\text { grupos de apoyo } \\
\text { desde la base } \\
\text { comunitaria } \\
\text { (Intervención). }\end{array}$ \\
\hline Apoyo evaluativo & $\begin{array}{l}\text { Autoevaluación - Grupo } \\
\text { de referencia } \\
\text { (Evaluación) }\end{array}$ & $\begin{array}{l}\text { Reflexión sobre la acción - } \\
\text { Comparación social } \\
\text { (Evaluación) }\end{array}$ & $\begin{array}{l}\text { Autoevaluación - Grupo de } \\
\text { referencia } \\
\text { (Evaluación) }\end{array}$ & $\begin{array}{l}\text { Reflexión sobre la } \\
\text { acción - } \\
\text { Comparación social } \\
\text { (Evaluación) }\end{array}$ \\
\hline Duración & $\begin{array}{l}6 \text { Meses } \\
\text { Enero } 2012\end{array}$ & $\begin{array}{l}6 \text { Meses } \\
\text { Julio } 2012\end{array}$ & $\begin{array}{l}18 \text { Meses } \\
\text { Enero } 2013\end{array}$ & $\begin{array}{l}12 \text { Meses } \\
\text { Julio } 2014\end{array}$ \\
\hline
\end{tabular}


Consideraciones éticas. La investigación corresponde al ejercicio fundamental de las disciplinas científicas en el ánimo de contribuir desde la academia a la explicación y comprensión de los fenómenos sociales que le lleven al hombre a potenciarse y reconocerse en su condición y naturaleza ineludiblemente humana. $\mathrm{Al}$ respecto señala el Código Deontológico de la Psicología reglamentado en la Ley 1090 de 2006 para el ejercicio de la profesión en Colombia: La Psicología es una ciencia y una profesión que estudia los procesos de desarrollo cognoscitivo, emocional y social del ser humano, reconociendo la complejidad de los grupos humanos y su contexto $^{19}$; en razón de ello la presente investigación, con el ánimo de contribuir en la construcción de escenarios vitales que permitan el desarrollo integral desde la dinamización del apoyo social, supuso el compromiso social al que estamos llamados los psicólogos en contexto.

En coherencia con el Título II, Disposiciones Generales, en el Artículo 2 literal 6, en el desarrollo de la investigación se informó de manera suficiente y pertinente a los participantes sobre el proceso, finalidad y destino de la información advirtiendo la libertad de participación dentro del desarrollo de la experiencia; a su vez, en coherencia con el literal 9 se salvaguardó la dignidad y el bienestar de las personas que participaron en conocimiento $\mathrm{y}$ acato de las normas legales y de los estándares profesionales que regulan la investigación ${ }^{19}$.

\section{Resultados}

Una de las principales preocupaciones de los equipos de intervención reside en la sostenibilidad de los procesos, una vez el equipo impulsor se retire del contexto. La investigación intervención concentró gran atención en el fortalecimiento del capital social desde la perspectiva del apoyo social que fomente un ambiente de confianza mutua, normas procedimentales claras y activación de redes de apoyo a fin de que se maximicen los niveles de colaboración social entre los diferentes grupos barriales como colectivo social proyectado a dar respuesta a los sistemas de apoyo comunitario.
El apoyo instrumental garantizó la participación de diversos agentes y actores comunitarios vinculados a redes naturales de apoyo, quienes articuladas con los agentes de interacción promovieron jornadas de atención dirigidas a la oferta de ayuda en situaciones relacionadas con la violencia intrafamiliar, salud sexual y reproductiva, consumos de sustancias psicoactivas, acompañamiento escolar y fortalecimiento de procesos comunitarios. (Tabla 2)

Tabla 2. Jornadas de apoyo instrumental y casos atendidos por agentes externos

\begin{tabular}{lccr}
\hline \multicolumn{1}{c}{ Ejes de atención } & $\begin{array}{c}\text { Número } \\
\text { de } \\
\text { jornadas }\end{array}$ & $\begin{array}{c}\text { Casos } \\
\text { atendidos }\end{array}$ & $\begin{array}{c}\text { Casos } \\
\text { remitidos } \\
\text { a ruta de } \\
\text { atención }\end{array}$ \\
\hline $\begin{array}{l}\text { Violencia } \\
\text { intrafamiliar }\end{array}$ & 10 & 142 & 85 \\
$\begin{array}{l}\text { Salud sexual y } \\
\text { reproductiva }\end{array}$ & 10 & 186 & 130 \\
$\begin{array}{l}\text { Consumo de SPA } \\
\begin{array}{l}\text { Acompañamiento } \\
\text { escolar }\end{array}\end{array}$ & 15 & 50 & 32 \\
$\begin{array}{l}\text { Fortalecimiento de } \\
\text { procesos } \\
\text { comunitarios y } \\
\text { seguridad } \\
\text { ciudadana. }\end{array}$ & 30 & 240 & \\
\hline
\end{tabular}

Desde los agentes locales se impulsaron escenarios de movilización donde las diversas redes naturales identificadas propusieron acciones de apoyo a miembros de la comunidad en situación de pobreza, marginalidad y enfermedad crónica.

El apoyo informacional generó procesos de reconocimiento de las rutas de atención institucionales; en materia de atención a víctimas de violencia sexual, atención a víctimas del conflicto armado, planificación familiar, acoso escolar, salud oral, apoyo socio jurídico y seguridad ciudadana. El seguimiento al uso de rutas de atención mostró un incremento del $2 \%$ al $28 \%$ en la utilización efectiva de las mismas, una vez se generó el proceso de socialización. Dentro del mismo eje se desarrollaron una serie de capacitaciones orientadas al reconocimiento de 
los servicios sociales, del estado social de derechos y el ejercicio de la ciudadanía; estos procesos de capacitación fortalecieron la dimensión organizativa de las redes de apoyo naturales, y una conciencia de exigibilidad de derechos en los participantes. El apoyo emocional evidenció el fortalecimiento socio-afectivo de los participantes dentro de sus redes naturales. Los reportes verbales en la autoevaluación del participante dentro de su grupo de referencia, muestra un crecimiento en los niveles de confianza y la comprensión empática, como también en los procesos comunicativos en relación de la consejería y la expresión de afecto, lo anterior traducido en un mejoramiento progresivo de las relaciones interpersonales y la instalación de agentes comunitarios como soporte emocional efectivos.

La estrategia de implementación de grupos de apoyo desde la base comunitaria, permitió la sustitución progresiva de los agentes generadores por los agentes locales, toda vez que miembros de la comunidad asumieron las responsabilidad de grupos de apoyo. En esta fase del proceso, dado los niveles de confianza, empatía y vínculos societales de los agentes locales con los demás participantes de los grupos de apoyo, la percepción de apoyo afectivo y la interacción social positiva se incrementaron del $40 \%$ al $67 \%$ a la finalización de la intervención.

En relación al apoyo evaluativo, se dieron los procesos de autoevaluación de agentes de acuerdo al grupo de referencia y procesos de reflexión colectiva sobre las estrategias y sus efectos en el entorno comunitario.
La oferta constante de información desde el seguimiento de casos, permitió los elementos informacionales para el fomento de la cultura de la autoevaluación que le permite al participante verbalizar la percepción de apoyo social de acuerdo a su grupo de referencia; el proceso de reflexión se sirvió del análisis de comparación social desde un línea base establecida con la propia comunidad. Como elementos relevantes identificados en los procesos de evaluación reflexión se reportó: a) El apoyo social incrementa el sentimiento de pertenencia del individuo al grupo, y de éste a la comunidad, b) El fortalecimiento de la autoestima del agente local, promueve la mejora sustantiva de la autoestima colectiva, c) Se evidencia un mejoramiento sustancial en los niveles de confianza, donde la mayor parte de participantes consideran que en situaciones de dificultad tienen la certeza de que van a contar con ayuda (apoyo social percibido).

En cuanto a los servicios sociales, el diagnóstico local de base favoreció la identificación progresiva de necesidades para la priorización de los servicios sociales que debían articularse a los centros de contacto, instalándose en el contexto programas de promoción del bienestar de la infancia y juventud, de protección y apoyo a la familia, de prevención y reinserción social de colectivos vinculados a conductas delictivas y consumo de drogas, de formación integral de agentes comunitarios de apoyo social, y de formación integral de personal para la salud y seguridad ciudadana. A continuación se muestra la articulación de las estrategias de interacción local como suministros de activación comunitaria con los servicios sociales. (Tabla 3 )

\section{Tabla 3. Articulación servicios sociales y estrategias de interacción local}

\begin{tabular}{|c|c|c|}
\hline Servicios sociales & Escenarios móviles & Centro de contacto \\
\hline Promoción del bienestar de la infancia y la juventud. & Espacios lúdico- comunitarios arte y deporte. & $\begin{array}{l}\text { Grupos asociativos de artes, } \\
\text { deporte y cultura. }\end{array}$ \\
\hline $\begin{array}{l}\text { Protección y Apoyo a la Familia. } \\
\text { Prevención y reinserción social de colectivos } \\
\text { sociales vinculados a conductas delictivas y } \\
\text { consumo de drogas. }\end{array}$ & $\begin{array}{l}\text { Implementación de grupos de apoyo sobre } \\
\text { violencia intrafamiliar, consumo de spa y } \\
\text { acompañamiento escolar. }\end{array}$ & $\begin{array}{l}\text { Valoración del apoyo social } \\
\text { percibido - Minimización del } \\
\text { riesgo a la violencia, el consumo } \\
\text { y la deserción escolar. }\end{array}$ \\
\hline $\begin{array}{l}\text { Formación integral de personal para la salud y } \\
\text { seguridad ciudadana }\end{array}$ & $\begin{array}{l}\text { Salud sexual y reproductiva, procesos } \\
\text { comunitarios y seguridad ciudadana. }\end{array}$ & $\begin{array}{l}\text { Fortalecimiento de la } \\
\text { pertenencia, autoestima } \\
\text { colectiva y percepción de } \\
\text { confianza. }\end{array}$ \\
\hline
\end{tabular}


Los espacios lúdico-comunitarios de arte y deporte constituyen una estrategia oportuna en el abordaje de procesos psicosociales con comunidades afrodescendientes y raizales. Dentro del desarrollo del escalamiento de objetivos se pudo identificar a la danza, el canto y el futbol como principales activos socioculturales para la adhesión comunitaria de poblaciones infanto-juveniles.

En la articulación entre apoyo social y servicios sociales, fue de suma relevancia la estrategia de grupos de apoyo implementados sobres redes comunitarias naturales, tales como hogares comunitarios, pastoral familiar, asociación de padres de familia lo que garantizó no solo la eficacia de la intervención sino el fortalecimiento de la cohesión comunitaria y la respuesta interinstitucional, logrando un impacto positivo en la disminución de las tasas de violencia intrafamiliar, consumo de SPA, y reinserción en conducta delictiva. Finalmente, la propuesta de investigación - intervención favoreció la integración de los servicios sociales en los centros de contacto como un sistema único de responsabilidad pública, donde las redes interinstitucionales garantizan la prestación del servicio acorde a las necesidades, dinámicas y procesos de apoyo y desarrollo de las comunidades vinculadas.

\section{Discusión}

En la actualidad las condiciones societales dadas las dinámicas económicas y culturales que permean las interacciones individuo - comunidad; presentan para el campo de las ciencias sociales aplicadas importantes retos, que lleven a controvertir las posiciones hegemónicas, excluyentes e ideologizantes que posicionan al individualismo como práctica social dominante; a lo que se suman los procesos de desorganización y disolución de redes de apoyo social, propio de sociedades industriales, donde son crecientes además problemas de índole psicosocial como la pobreza, delincuencia y estrés ${ }^{20}$.

El estudio del apoyo social constituye un campo conceptual que progresivamente ha venido ganando espacios en su articulación con la salud y el desarrollo comunitario ${ }^{20}$. Es así como el presente artículo da cuenta de su eficacia a través de la implementación de estrategias de escenarios móviles y centros de contacto comunitario que garanticen la accesibilidad de los habitantes de la Comuna 5 de Buenaventura a los servicios comunitarios, y promuevan el desarrollo, desde el fortalecimiento de redes de apoyo como capacidad local instalada.

De acuerdo con San Martín \& Barra ${ }^{21}$ el apoyo emocional referido a conductas de cuidado, permite dentro de centros de contacto, que los habitantes en espacios comunales puedan compartir pensamientos y experiencias personales; mientras el apoyo informacional favorece la apropiación y diseminación de informaciones pertinentes para el afrontamiento colectivo de las demandas del entorno. De allí, la relación positiva entre escenarios móviles y apoyo social, puesto que fomenta espacios de movilización de los actores locales, promueve emociones positivas, minimizando tanto los niveles de estrés comunitario como la percepción del conflicto ${ }^{22}$.

El apoyo instrumental abordado desde los centros de contacto comunitario, permite la entrega efectiva de ayuda material, y articula la prestación de servicios sociales; lo anterior, de acuerdo con Valois et al., ${ }^{23}$ para quien la percepción del apoyo social en centros de contacto, disminuye los factores de riesgo y promueve cambios importantes en la autoestima colectiva, lo que mejora los niveles de apoyo social y cooperación en la comunidad, de allí la importancia del desarrollo en los escenarios móviles de grupos asociativos de artes, deporte y cultura que vinculen activamente a los beneficiarios dentro de la gestión local.

En cuanto a la prestación de servicios sociales, entornos comunitarios que promuevan el contacto y la participación permiten el establecimiento de vínculos entre usuarios y profesionales ${ }^{24}$, garantizando una mayor adhesión de la comunidad, y una mejora sustancial en los niveles de satisfacción de los ciudadanos frente a la prestación de servicios. 
Para los casos de violencia intrafamiliar, consumo de spa y acoso escolar los centros de contacto establecen alianzas terapéuticas y acuerdos simbólicos interventor - usuario, que no sólo promueven habilidades de afrontamiento, a su vez restablecen el funcionamiento social del individuo dentro de la comunidad.

Las diferentes valoraciones del apoyo social percibido frente a los escenarios móviles y sus redes de apoyo socio-culturales; muestra como la vinculación directa de los agentes comunitarios como agentes de ayuda, incrementa de manera sustancial la percepción de apoyo, y favorece una mayor adherencia de la comunidad a la estrategia de desarrollo; al respecto, Colvin et al., ${ }^{25}$ sostienen que las redes organizadas de relaciones sociales de carácter barrial o societal son eficaces en la satisfacción de necesidades emocionales o instrumentales, siendo mayores los niveles de apoyo social percibido en comunidades donde agentes locales se vinculan al desarrollo de las estrategias, lo anterior, por cuanto los participantes perciben más cercano el apoyo disponible si tienen altos niveles de confianza con el agente de ayuda ${ }^{26}$.

En el contexto comunitario los grupos de apoyo logran trastocar patrones relacionales lesivos a los procesos de convivencia, y favorecen una percepción positiva del ambiente y su cuidado ${ }^{22}$. Sin duda el apoyo social articulado a centros de contacto y escenarios móviles, no sólo acerca el servicio social a la comunidad, también promueve en ella la capacidad de afrontamiento, proporcionando condiciones para enfrentar los problemas locales manteniendo en sus habitantes un ajuste psicológico adecuado.

Son evidentes los efectos beneficiosos del apoyo social desde de los centros de contacto, de hecho, Mendez \& Barra $^{27}$ plantean la existencia de una relación positiva entre bienestar individual y relaciones sociales. De allí que sean evidentes a lo largo de la investigación-intervención, cambios tanto en el auto-concepto, control personal y empatía de los participantes ${ }^{28}$.

En este contexto, pensar los escenarios móviles, como una experiencia significativa en la articulación del apoyo social, localiza el interés e intencionalidad de la intervención en medio de la comunidad, en el seno de las redes de apoyo. En ese propósito, es la participación comunitaria, el principal componente de vinculación y adhesión de la comunidad no sólo en el desarrollo de acciones de cambio, sino en la toma de decisiones colectivas donde se direcciona el curso de la intervención ${ }^{12}$, situación cada vez más apremiante en el contexto del pacifico colombiano, donde los problemas psicosociales muestran mayor dificultad de ser mitigados sin intervenciones directas. En efecto, la estrategia de escenarios móviles y centros de contacto constituye una propuesta innovadora en el fortalecimiento de la articulación de las Instituciones con las comunidades, potenciando el desarrollo de recursos locales desde redes mutuales de apoyo, la participación activa y la organización social donde se garantice la disponibilidad del capital social y la adherencia del individuo al proceso ${ }^{10}$.

Los servicios sociales se articulan de manera progresiva a los centros de contacto generando un tejido estructural y colaborativo, al proveer al contexto de suministros técnicos, procedimentales y de talento humano; mientras que los escenarios móviles se convierten en una herramienta de orientación del conjunto de la población de ámbito local- barrial a los diversos procesos institucionales que les garanticen mejor calidad de vida, apoyo social eficaz y accesibilidad a los servicios institucionales ${ }^{13}$.

En efecto, la estrategia de escenarios móviles y centro de contacto se convierte en una importante herramienta en el fomento del apoyo social, donde se articule la realidad de los contextos con la oferta y acción interinstitucional de programas que resuelvan en el contexto los problemas sociales, asegurando la universalidad y eficacia de los programas gubernamentales de bienestar social28. Asimismo, la participación activa de los destinatarios en el diseño, planificación y evaluación de los marcos y escenarios propuestos, garantiza la apropiación de los procesos de intervención por las comunidades beneficiarias. 
En la actualidad existe un nuevo contexto social, formas y valores culturales que requieren de nuevas políticas sociales, que integren a los agentes locales en la ejecución de estrategias de intervención en el campo del desarrollo cultural, educativo y de acción social ${ }^{29}$. Para Tobon ${ }^{30}$ en relación a los recursos locales, las comunidades, grupos y personas desarrollan prácticas protectoras, sin embargo, no gestionan acciones de prevención que minimicen el riesgo del problema psicosocial; de allí, la necesidad de integrar procesos de capacitación en servicios sociales, derechos y ciudadanía, a fin de fomentar en el capital social local la apropiación del herramientas prácticas y el desarrollo de habilidades, confiriendo nuevas capacidades comunitarias necesarias para la activación social y la movilización de recursos relacionales y de apoyo comunitario.

Para Colmenares ${ }^{8}$ es sabido, que la principal contribución de los escenarios móviles en la reducción de factores de riesgo y la promoción del apoyo social, está asociada a la participación directa de las organizaciones comunitarias, redes naturales e individuos en la solución de sus necesidades; razón por la cual cabe destacar la relevancia de las redes de acción y gestión comunitaria circunscritas a los centros de contacto comunitario, desde donde se proyecta la agenda comunitaria y se buscan soluciones colectivas a los problemas; es así, como el desarrollo de escenarios móviles fortalece la pertenencia, autoestima colectiva, percepción de confianza y honestidad gubernamental de los usuarios frente a los prestadores de servicio.

Dentro de los escenarios móviles, las jornadas de atención y movilización del apoyo comunitario, restablecen la prestación de servicios sociales y motivan el desarrollo de estrategias de ayuda al interior de las redes informales, frente a problemas como el conflicto y la violencia. De acuerdo a Lopata ${ }^{31}$ se podría concebir a los escenarios moviles y centros de contacto como un sistema de apoyo primario, desde el cual se provee servicios sociales, apoyo social emocional y se fomentan los grupos de ayuda y redes naturales de apoyo; donde las redes y grupos de ayuda son instancias mediadoras entre el usuario y la percepción positiva de apoyo ${ }^{32}$ en sus dimensiones instrumental, emocional, informacional y evaluativa; percepción que logra mejorar el conjunto de relaciones interpersonales e institucionales, y le permite al individuo y comunidades mejorar su bienestar material, físico y emocional ${ }^{33}$.

La ejecución de escenarios móviles y centros de contacto como experiencia significativa en la dinamización del apoyo social desde el recurso local, favorece el intercambio y la reciprocidad agente - comunidad, y permite mayor eficacia en la gestión de la información y la recepción de ayuda, a fin de incrementar el bienestar tanto individual como comunitario.

Al respecto, en coherencia con los postulados de Gottlieb ${ }^{6}$ sobre los cinco niveles o estructuras del apoyo social, la estrategia de escenarios móviles desde acciones locales intencionales, favorece la modificación del ambiente físico y sociocultural en el que se desenvuelve la persona, propiciando en los intercambios barriales cambios en las funciones de los lazos sociales, desde aspectos como las normas, roles y responsabilidades locales en los procesos comunitarios; mientras el centro de contacto enfocado en la transformación de la estructura comunitaria promueve la confianza pública en las fuentes informales de ayuda y estimula expresiones de apoyo en la vida cotidiana, canalizando el apoyo socioafectivo, informacional e instrumental de los participantes dentro de sus redes naturales $y$ de las instituciones prestadoras de servicios sociales.

\section{Conclusiones}

En la democratización como acceso universal y participativo de la ciudadanía a los servicios sociales, los centros de contacto se convierten en una herramienta efectiva en la identificación y fortalecimiento de las capacidades comunitarias, desde el fomento de la participación de los agentes locales en la toma de decisiones, la gestión de estrategias locales y el acceso progresivo a cargos de representación, la administración de recursos públicos y la movilización de procesos comunitarios. En la 
articulación efectiva de los escenarios móviles y centros de contacto se debe garantizar la vinculación directa de los destinatarios en la toma de decisiones, de forma que progresivamente los agentes locales asuman mayores responsabilidades dentro del proceso, situación que garantiza un capital social instalado sobre el desarrollo de la capacidad comunitaria, por encima del suministro de activación externo.

Las estrategias de acción motivacionales para ser efectivas deben valorar las condiciones culturales, sociales y territoriales del entorno de interacción 46 , razón por la cual, es oportuno en el trabajo con comunidades afrodescendientes construir la ruta de participación y movilización desde la promoción activos socioculturales. Los escenarios móviles constituyen una importante herramienta para la gestión de apoyo instrumental al movilizar las estructuras sociales en el marco de la solidaridad comunitaria y proveer desde la esfera local ayuda eficiente y efectiva. En este contexto, la estrategia de escenarios móviles y centros de contacto se convierte en una innovadora alternativa para la promoción de la participación ciudadana en la prestación dinamización del apoyo social, garantizando no sólo mayor accesibilidad y equidad en la prestación de servicios, sino también el desarrollo del capital social en torno a las capacidades comunitarias locales.

La dinamización de las redes naturales en grupos de ayuda, articulados a escenarios móviles y centros de contacto, permite proporcionar al individuo desde su base el acceso a los servicios sociales (redes formales de apoyo) y vínculos de colaboración - reciprocidad desde las esferas societales en sus grupos de referencia, familia, amigos, ambiente laboral (redes informales de apoyo). De este modo, la estrategia de intervención es efectiva en relación al fortalecimiento de las relaciones sociales y la percepción de ayuda en las mismas.

Es necesario el desarrollo de acciones que promuevan el apoyo social con un enfoque multinivel, en el nivel uno, el agente interventor debe promover procesos de integración y reconocimiento de los agentes locales con los procesos comunitarios; en el segundo nivel, es necesario establecer relaciones de calidad, donde se enfatice sobre las necesidades de afiliación y reconocimiento y se active en la comunidad sentimientos de identidad y pertenencia, en el nivel tres, debe darse claridad frente a las fuentes de información y el acceso a agentes y grupos de apoyo, de manera que el individuo pueda mejorar la percepción de ayuda percibida, maximizando los niveles de confianza en los agentes locales e instituciones; finalmente, en el nivel cuatro, es necesario valorar la cantidad de casos donde existe ayuda efectiva, y promover la autogeneración de fuentes de ayuda, condición que da sostenibilidad comunitaria al proceso.

Finalmente, el apoyo social permite a las comunidades en ambientes sociales de pobreza, violencia y desintegración promover la autoestima colectiva y la solidaridad efectiva, como atenuantes de estresores sociales marcados por el individualismo y la exclusión social. Los grupos de apoyo permitieron valorar a los estresores como menos amenazantes, y visualizar nuevas experiencias de afrontamiento. De este modo el modelo de apoyo social en la Comuna 5 de Buenaventura fue un herramienta de intervención local útil, entre tanto proporcionó beneficios significativos tanto al individuo, los grupos naturales como las redes informales, generando en los individuos mayor estabilidad, control de procesos, autoeficacia y potenciación de recursos personales y sociales.

Conflicto de intereses: Ninguno declarado por autores.

\section{Referencias}

1. Colombia. Departamento Administrativo Nacional de Estadística - DANE. Censo Nacional de Población 2012. Disponible

en: http://190.25.231.242/cgibin/RpWebEngine.eje/P ortalAction?\&MODE=MAIN\&BASE=CG2005AMPLIADO \&MAIN=WebServerMain.inl

2. Carvajal C. Etnoeducación y realidades del pacífico colombiano. Bogotá: FCE; 2004.

3. Fundación Rostros y Huellas del Sentir Humano. Situación de derechos humanos y desplazamiento forzado en la Comuna 5 Buenaventura. Documento sin publicar, archivo Fundación; 2010.

4. Perea, J. Prácticas sociales y violencia. Cali: Rev. Libertaria. 2011;2(6):45-56. 
5. Barrón A, Sánchez E. Estructura social, apoyo social y salud mental. Psicothema. 2013; 13:17-23.

6. Gottlieb, B. Support interventions: A tipology and agenda for research. In S. W. Duck. Hankbook of personal relationships. New York: Wiley and sons; 1983.

7. Max-Neef, M. La economía descalza. Buenos Aires: ED. Norman, 3 Ed; 1986.

8. Colmenares, J. Organización comunitaria y derechos humanos. Caracas: ED. Libertador; 2012.

9. Durá E, Gárcés J. La teoría del apoyo social y sus implicaciones para el ajuste psicosocial de los enfermos oncológicos. Revista de Psicología Social 1991; 6(2), 257-271.

10. Herrera M. Intervención comunitaria, perspectiva social. BsAs: Santillana; 2013.

11. Musito G Herrero J, Cantera L, Montenegro M. Introducción a la psicología Comunitaria. Barcelona: Ed. UCO; 2004.

12. Narváez JH. Escenarios móviles y centros de contacto, una experiencia significativa para el desarrollo comunitario de los servicios sociales. Revista CES Psicología 2015; 8(2), 122-137.

13. Narváez J. Modelo de transitividad aplicado a la gestión de la salud mental comunitaria. Rev Univ. Salud. 2014; No. 16(1): 67 - 79.

14. Fals-Borda O. Conocimiento y poder popular. Bogotá: Siglo XXI; 1985.

15. López de Ceballos P. Un método para la investigaciónacción participativa. Madrid: ED. Popular; 1987.

16. Ahumada M, Anton BM, Peccinetti MV. Investigación Acción Participativa en Psicología. San Martín: Rev. Enfoques. 2012; 24(2):23-52

17. Ander-Egg, E. Métodos y técnicas de investigación social: Técnicas para la recogida de datos e información. Buenos Aires: Lumen; 2003.

18. Montes A. Simbolismo y poder: Un estudio antropológico sobre compadrazgo y priotazgo en una comunidad andina. Barcelona: Editorial Anthropos; 1989.

19. Colombia. Ley 1090. Código deontológico y bioético y otras disposiciones. Bogotá: Pontificia Universidad Javeriana; 2006.

20. García E, Herrero JY, Musitu G. Evaluación de recursos y estresores psicosociales en la comunidad. Madrid: Síntesis; 2002.

21. San Martín J, Barra E. Autoestima, Apoyo Social y Satisfacción Vital en Adolescentes. Terapia psicológica, 2013, 31(3), 287-291.

22. Barra E. Apoyo social, estrés y salud. Revista Psicología y Salud, 2004 Vol. 14, Núm. 2: p. 237-243.

23. Valois RF, Zullig KJ, Huebner ES, Drane JW. Youth developmental assets and perceived life satisfaction: Is there a relationship? Applied Research Quality of Life, 2009; 4, 315-331.

24. Leibe M, Kallert T. Integración social y calidad de vida de los pacientes esquizofrénicos en tipos de asistencia complementaria diferentes. Eur Psychiatry Ed Esp, 2001; 8: 93-104.

25. Colvin M, Cullen F, Vander-Ven T. Coercion, social support, and crime: An emerging theoretical consensus. Criminology, 2002; 40, 19-42.
26. Gracia E. El apoyo social en la intervención comunitaria. Buenos Aires: Paidós; 1997.

27. Méndez P, Barra E. Apoyo Social Percibido en Adolescentes Infractores de Ley y no Infractores. Psykhe, 2008, 17(1), 59-64.

28. Gonzales L. Acción social y participación ciudadana. Madrid: ED. Santillana; 2013.

29. López F, León L. La animación sociocultural como contribución a la construcción de la identidad comunitaria. Sevilla: Cuestiones pedagógicas, Rev. Ciencias de la Educación, 2002; 16: 139 - 150.

30. Tobón-Correa O. El autocuidado una habilidad para vivir. 2010; recuperado el 03 de abril de 2016, disponible en: http://promocionsalud.ucaldas.edu.co/downloads/Rev ista\%208_5.pdf

31. Lopata H. Women ad wodows: suport systems. Nueva York.: Elsevier; 1975.

32. Cobb, S. Social support as a moderator of life stress. Psychosomatic Medicine,1976; 38: 300-314.

33. Guzmán J, Huenchuan S, Montes De Oca V. Redes de apoyo social de personas mayores: marco teórico conceptual. Ponencia presentada en el Simposio Viejos y Viejas. Participación, Ciudadanía e Inclusión Social. Santiago de Chile; 2003. 